\title{
Erratum to: Bioinformatics and Biomedical Engineering
}

\author{
Ignacio Rojas $^{(\bowtie)}$ and Francisco Ortuño \\ Universidad de Granada, Granada, Spain
}

\section{Erratum to: \\ Chapter "An Automatic and Intelligent System for Integrated Healthcare Processes Management" in: I. Rojas and F. Ortuño (Eds.): Bioinformatics and Biomedical Engineering,}

Part II, LNBI 10209, https://doi.org/10.1007/978-3-319-56154-7_55

The initially published versions of Acknowledgements section were incorrect. This is the correct version:

Acknowledgements. This research has been supported by the Pololas project (TIN2016-76956-C3-2-R) and by the SoftPLM Network (TIN2015-71938-REDT) of the Spanish the Ministry of Economy and Competitiveness.

\section{Erratum to:}

Chapter "A Microcontroller Based System for Controlling Patient Respiratory Guidelines" in: I. Rojas and F. Ortuño (Eds.): Bioinformatics and Biomedical Engineering, Part II, LNBI 10209, https://doi.org/10.1007/978-3-319-56154-7_56

The initially published versions of Acknowledgements section were incorrect. This is the correct version:

Acknowledgements. This research has been supported by the Pololas project (TIN2016-76956-C3-2-R) and by the SoftPLM Network (TIN2015-71938-REDT) of the Spanish the Ministry of Economy and Competitiveness.

The updated online version of this book can be found at https://doi.org/10.1007/978-3-319-56154-7 https://doi.org/10.1007/978-3-319-56154-7_55 https://doi.org/10.1007/978-3-319-56154-7_56 